\title{
Improved Production of Sublancin 168 Biosynthesized by Bacillus subtilis 168 Using Chemometric Methodology and Statistical Experimental Designs
}

\author{
Shengyue Ji, Weili Li, Haiyun Xin, Shan Wang, and Binyun Cao \\ College of Animal Science and Technology, Northwest A\&F University, 22 Xinong Road, Yangling, Shaanxi 712100, China \\ Correspondence should be addressed to Binyun Cao; jiziqing2010@163.com
}

Received 17 October 2014; Revised 31 December 2014; Accepted 5 January 2015

Academic Editor: Victor Fedorenko

Copyright (C) 2015 Shengyue Ji et al. This is an open access article distributed under the Creative Commons Attribution License, which permits unrestricted use, distribution, and reproduction in any medium, provided the original work is properly cited.

\begin{abstract}
Sublancin 168, as a distinct S-linked antimicrobial glycopeptide produced by Bacillus subtilis 168, is effective in killing specific microorganisms. However, the reported yield of sublancin 168 is at a low level of no more than $60 \mathrm{mg}$ from $1 \mathrm{~L}$ fermentation culture of B. subtilis 168 by using the method in the literature. Thus optimization of fermentation condition for efficiently producing sublancin 168 is required. Here, Box-Behnken design was used to determine the optimal combination of three fermentation parameters, namely, corn powder, soybean meal, and temperature that were identified previously by Plackett-Burman design and the steepest ascent experiment. Subsequently, based on the response surface methodology, the quadratic regression model for optimally producing sublancin 168 was developed, and the optimal combination of culture parameters for maximum sublancin 168 production of $129.72 \mathrm{mg} / \mathrm{L}$ was determined as corn powder $28.49 \mathrm{~g} / \mathrm{L}$, soybean meal $22.99 \mathrm{~g} / \mathrm{L}$, and incubation temperature $30.8^{\circ} \mathrm{C}$. The results showed that sublancin 168 production obtained experimentally was coincident with predicted value of $125.88 \mathrm{mg} / \mathrm{L}$, and the developed model was proved to be adequate, and the aim of efficiently producing sublancin 168 was achieved.
\end{abstract}

\section{Introduction}

Sublancin 168 is a novel and distinct S-linked bacteriocin glycopeptide consisting of 37 amino acids and is produced by Bacillus subtilis 168 strains $[1,2]$. Based on its potent antimicrobial activity in inhibiting special Gram-positive bacteria, including B. megaterium, B. subtilis 6633, the pathogenic microbes Streptococcus pyogenes, and Staphylococcus aureus [1], this antimicrobial peptide could be used in a wide range of commercial applications, such as agriculture, cosmetics, and pharmaceutical field. However, the host strain was cultured with a medium used for producing subtilin $[1,3]$, which contains (per liter) sucrose $20 \mathrm{~g}$, citric acid $11.7 \mathrm{~g}$, $\mathrm{Na}_{2} \mathrm{SO}_{4} 4 \mathrm{~g},\left(\mathrm{NH}_{4}\right)_{2} \mathrm{HPO}_{4} 4.2 \mathrm{~g}$, yeast extract $5 \mathrm{~g}, 100 \mathrm{~mL}$ of a salt mixture $\left(\mathrm{KCl} 7.62 \mathrm{~g}, \mathrm{MgCl}_{2} \cdot 6 \mathrm{H}_{2} \mathrm{O} 4.18 \mathrm{~g}, \mathrm{MnCl}_{2} \cdot 4 \mathrm{H}_{2} \mathrm{O}\right.$ $0.543 \mathrm{~g}, \mathrm{FeCl}_{3} \cdot 6 \mathrm{H}_{2} \mathrm{O} 0.49 \mathrm{~g}$, and $\mathrm{ZnCl}_{2} 0.208 \mathrm{~g}$ in $1000 \mathrm{~mL}$ $\mathrm{H}_{2} \mathrm{O}$ ), and sufficient $\mathrm{NH}_{4} \mathrm{OH}$ to bring the $\mathrm{pH}$ to 6.8-6.9. As a result of complex medium ingredients and nonspecialized medium for producing sublancin 168, some events of the pinkish-brown color, fruity odor, and $\mathrm{pH}$ value near 6 that accompanying with good sublancin 168 production did not always occur, whereupon the production of sublancin 168 was usually at a low level of no more than $60 \mathrm{mg}$ from $1 \mathrm{~L}$ bacterial culture [1]. The low yield of sublancin 168 has constrained its commercial application, and the optimization of fermentation conditions is required to allow for efficient production of sublancin 168.

Recently, there has been an increasing interest in response surface methodology processes for improving productivities of natural bioactive agents $[4,5]$. Several bioactive proteins, such as eicosapentaenoic acid [6], tostadin [7], and antimicrobial compounds $[8,9]$, produced by bacteria strains optimized through response surface methodology have been recorded. However, literature is lacking cultivation optimization of sublancin 168 produced by B. subtilis 168 using chemometric and statistical methodology.

The aim of this current work was to efficiently produce sublancin 168 via optimizing the variables of medium compositions and culture conditions by using statistical tools in shake-flasks. In the first step, Plackett-Burman design as an 
effective technique was used to screen remarkable variables. Subsequently, the steepest ascent was utilized to approach the optimal region. At last, Box-Behnken design and response surface analysis were employed to ascertain the optimum levels of the factors which significantly effect sublancin 168 productions. In this study, the sublancin 168 production at a high level is achieved through adopting chemometric and statistical methodology.

\section{Materials and Methods}

2.1. Materials. Yeast extract and tryptone were purchased from Difco (Detroit, USA). Corn powder and soybean meal were purchased from Sinopharm Chemical Reagent Beijing Co., Ltd. (China) and Shandong Litong Biotechnology Co., Ltd. (China), respectively, and were passed through 60-mesh sieve. Other chemicals used were of chemical grade.

2.2. Bacteria Strains and Fermentation Condition. B. subtilis 168 (ATCC 27370), the producer of sublancin 168, was utilized in the current work [1]. The strains were maintained on Luria-Bertani medium (LB) agar slant with the following composition (g/L): tryptone 10.0, yeast extract 5.0, $\mathrm{NaCl} 5.0$, and agar 18.0 with the $\mathrm{pH}$ value to 7.0. After culturing at $37^{\circ} \mathrm{C}$ for 28 hours, the slants were subcultured once a month and stored at $4^{\circ} \mathrm{C}$.

Seed culture of B. subtilis 168 was prepared by culturing bacterial strains in a $250 \mathrm{~mL}$ flask containing $50 \mathrm{~mL}$ LB liquid medium at $37^{\circ} \mathrm{C}, 225 \mathrm{rpm}$ for 12 hours. Subsequently, $1 \mathrm{~mL}$ prepared seed culture was inoculated into $250 \mathrm{~mL}$ flask containing $50 \mathrm{~mL}$ culture medium (g/L): peptone 10.0, starch 15.0, $\mathrm{KH}_{2} \mathrm{PO}_{4} 4.0$, and $\left(\mathrm{NH}_{4}\right)_{2} \mathrm{SO}_{4} 4.0$, and the $\mathrm{pH}$ was adjusted to 7.0, and furtherly cultured for 48 hours. After fermentation, the culture supernatant was harvested by removing the cells and the debris through centrifugation $10,000 \mathrm{~g}$ for $5 \mathrm{~min}$. Each test was repeated three times and the average of sublancin 168 concentration was taken as the response.

2.3. Screen of Carbon and Nitrogen Sources. The optimal nitrogen and carbon sources effecting sublancin 168 production were screened by one variable at a time (OVAT) approach. The evaluations of different simple and complex nitrogen (yeast extract, peptone, soybean meal, urea, and $\left(\mathrm{NH}_{4}\right)_{2} \mathrm{SO}_{4}$ ) and carbon sources (corn powder, glycerol, sucrose, lactose, starch, maltose, and glucose) on sublancin 168 production were performed one by one (Table 1 ). The above different nitrogen sources $(5 \mathrm{~g} / \mathrm{L})$ and carbon sources $(10 \mathrm{~g} / \mathrm{L})$ instead of peptone and starch were taken into the culture procedure as described above.

2.4. Plackett-Burman Design. The Plackett-Burman design is a powerful tool for rapidly screening and determining the important variables that has significant influence on the production response. This method was very useful for picking the most important factors from a long list of candidate factors [10]. In this work, different cultivation parameters (inoculum size, initial $\mathrm{pH}$, incubation temperature, and incubation time)
TABLE 1: Effects of different carbon sources and nitrogen sources on the yield of sublancin 168 .

\begin{tabular}{lccc}
\hline \multicolumn{2}{c}{ Carbon sources } & \multicolumn{2}{c}{ Nitrogen sources } \\
Sources & Yield $(\mathrm{mg} / \mathrm{L})$ & Sources & Yield $(\mathrm{mg} / \mathrm{L})$ \\
\hline Corn powder & $67.66 \pm 3.56$ & Yeast extract & $28.89 \pm 4.72$ \\
Glycerol & $28.96 \pm 4.39$ & Peptone & $50.11 \pm 4.56$ \\
Sucrose & $30.55 \pm 4.90$ & Soybean meal & $58.40 \pm 5.33$ \\
Lactose & $29.61 \pm 5.03$ & Urea & $8.72 \pm 3.18$ \\
Starch & $50.65 \pm 4.85$ & $\left(\mathrm{NH}_{4}\right)_{2} \mathrm{SO}_{4}$ & $32.93 \pm 2.10$ \\
Maltose & $28.26 \pm 5.10$ & & \\
Glucose & $21.40 \pm 5.49$ & & \\
\hline
\end{tabular}

Each experiment was repeated three times, and all of the data were expressed as means \pm standard deviations.

and medium components (peptone, soybean meal, starch, corn powder, $\mathrm{KH}_{2} \mathrm{PO}_{4}$, and $\left.\left(\mathrm{NH}_{4}\right)_{2} \mathrm{SO}_{4}\right)$ were evaluated utilizing Plackett-Burman design to identify the important factors influencing sublancin 168 production greatly. Each factor at two levels was examined based on Plackett-Burman factorial design: -1 and +1 for low and high level, respectively [11]. On the preliminary study, it was found that the optimal temperature for producing sublancin 168 by B. subtilis 168 was at $32^{\circ} \mathrm{C}$. Therefore, in Plackett-Burman experiment, the culture temperature test level was set between $30^{\circ} \mathrm{C}$ and $34^{\circ} \mathrm{C}$. Table 2 illustrates the factors under investigation and the levels of each factor setting in the experimental design. Response values were determined based on sublancin 168 productions.

The Plackett-Burman design was established by SAS software package (version 9.1.3, SAS Institute Inc., Cary, NC, USA) in terms of the following first-order model:

$$
Y=\beta_{0}+\sum_{i=1}^{k} \beta_{i} X_{i}
$$

where $Y$ refers to the response (i.e., sublancin 168 production) and $\beta_{0}, \beta_{i}, X_{i}$, and $k$ represent the constant, the linear coefficient, the level of the independent variables, and the number of involved variables, respectively.

In addition to the variables of real interest, the PlackettBurman design considers insignificant dummy variables, which are introduced to evaluate the experimental error and the variance of the first-order model. In this work, 10 variables were checked in 20 trials (Table 3). Every trial was performed three times, and the average sublancin 168 production was applied as the response variable. Regression analysis determined the variables that had a significant effect $(P<$ 0.05 ) on sublancin 168 production, and these variables were subsequently evaluated in further optimization experiments.

2.5. Steepest Ascent Method. In general, some variations of the optimum culture condition for the system exist between the actual optimum and the initial estimate. In such case, the single steepest ascent experiment was performed to optimize the variables influenced sublancin 168 production significantly [12]. 
TABLE 2: Variables and test levels for Plackett-Burman experiment.

\begin{tabular}{lccccccc}
\hline \multirow{2}{*}{ Number } & Variables & \multicolumn{2}{c}{ Code levels } & Estimate & t-value & $P$ value & Significance \\
& & -1 & 1 & & & \\
\hline$X_{1}$ & Peptone $(\mathrm{g} / \mathrm{L})$ & 8 & 12 & 2.33 & 1.01 & 0.3851 \\
$X_{2}$ & Corn powder $(\mathrm{g} / \mathrm{L})$ & 20 & 30 & 27.20 & 11.83 & 0.0013 \\
$X_{3}$ & Starch $(\mathrm{g} / \mathrm{L})$ & 10 & 20 & 3.19 & 1.39 & 0.2594 \\
$X_{4}$ & Soybean meal $(\mathrm{g} / \mathrm{L})$ & 24 & 36 & 21.09 & 9.17 & 0.0027 \\
$X_{5}$ & $\mathrm{KH}_{2} \mathrm{PO}_{4}(\mathrm{~g} / \mathrm{L})$ & 3 & 6 & 3.21 & 1.39 & 0.2575 \\
$X_{6}$ & $\left(\mathrm{NH}_{4}\right)_{2} \mathrm{SO}_{4}(\mathrm{~g} / \mathrm{L})$ & 3 & 6 & -0.96 & -0.42 & 0.7038 \\
$X_{7}$ & Incubation temperature $\left({ }^{\circ} \mathrm{C}\right)$ & 28 & 34 & 11.11 & 4.83 & 0.0169 \\
$X_{8}$ & Initial $\mathrm{pH}$ & 6.5 & 8.5 & -1.91 & -0.83 & 0.0466 \\
$X_{9}$ & Incubation time $(\mathrm{h})$ & 28 & 40 & 0.38 & 0.17 & 0.8786 \\
$X_{10}$ & Inoculum size $(\%)$ & 1 & 3 & -2.34 & -1.01 & 0.3834 \\
\hline
\end{tabular}

* indicates model terms are significant.

TABle 3: Experimental design and results of the Plackett-Burman design.

\begin{tabular}{|c|c|c|c|c|c|c|c|c|c|c|c|c|}
\hline \multirow{2}{*}{ Trials } & \multicolumn{10}{|c|}{ Variable levels } & \multicolumn{2}{|c|}{ Yield (mg/L) } \\
\hline & $X_{1}$ & $X_{2}$ & $X_{3}$ & $X_{4}$ & $X_{5}$ & $X_{6}$ & $X_{7}$ & $X_{8}$ & $X_{9}$ & $X_{10}$ & Observed & Predicted \\
\hline 1 & 1 & -1 & 1 & 1 & -1 & -1 & -1 & -1 & 1 & -1 & $88.38 \pm 3.84$ & 90.91 \\
\hline 2 & 1 & 1 & -1 & 1 & 1 & -1 & -1 & -1 & -1 & 1 & $117.96 \pm 2.37$ & 115.41 \\
\hline 3 & -1 & 1 & 1 & -1 & 1 & 1 & -1 & -1 & -1 & -1 & $97.42 \pm 6.78$ & 96.55 \\
\hline 4 & -1 & -1 & 1 & 1 & -1 & 1 & 1 & -1 & -1 & -1 & $101.79 \pm 3.41$ & 98.33 \\
\hline 5 & 1 & -1 & -1 & 1 & 1 & -1 & 1 & 1 & -1 & -1 & $102.43 \pm 3.74$ & 99.73 \\
\hline 6 & 1 & 1 & -1 & -1 & 1 & 1 & -1 & 1 & 1 & -1 & $92.45 \pm 3.96$ & 94.17 \\
\hline 7 & 1 & 1 & 1 & -1 & -1 & 1 & 1 & -1 & 1 & 1 & $107.47 \pm 9.89$ & 104.83 \\
\hline 8 & 1 & 1 & 1 & 1 & -1 & -1 & 1 & 1 & -1 & 1 & $121.38 \pm 2.26$ & 124.57 \\
\hline 9 & -1 & 1 & 1 & 1 & 1 & -1 & -1 & 1 & 1 & -1 & $119.62 \pm 2.27$ & 117.05 \\
\hline 10 & 1 & -1 & 1 & 1 & 1 & 1 & -1 & -1 & 1 & 1 & $88.76 \pm 5.85$ & 90.81 \\
\hline 11 & -1 & 1 & -1 & 1 & 1 & 1 & 1 & -1 & -1 & 1 & $119.63 \pm 5.56$ & 123.21 \\
\hline 12 & 1 & -1 & 1 & -1 & 1 & 1 & 1 & 1 & -1 & -1 & $79.81 \pm 4.77$ & 80.87 \\
\hline 13 & -1 & 1 & -1 & 1 & -1 & 1 & 1 & 1 & 1 & -1 & $118.85 \pm 5.84$ & 120.81 \\
\hline 14 & -1 & -1 & 1 & -1 & 1 & -1 & 1 & 1 & 1 & 1 & $76.33 \pm 3.15$ & 77.53 \\
\hline 15 & -1 & -1 & -1 & 1 & -1 & 1 & -1 & 1 & 1 & 1 & $82.21 \pm 2.79$ & 80.17 \\
\hline 16 & -1 & -1 & -1 & -1 & 1 & -1 & 1 & -1 & 1 & 1 & $77.19 \pm 4.41$ & 76.27 \\
\hline 17 & 1 & -1 & -1 & -1 & -1 & 1 & -1 & 1 & -1 & 1 & $62.37 \pm 5.76$ & 61.05 \\
\hline 18 & 1 & 1 & -1 & -1 & -1 & -1 & 1 & -1 & 1 & -1 & $106.22 \pm 7.25$ & 104.95 \\
\hline 19 & -1 & 1 & 1 & -1 & -1 & -1 & -1 & 1 & -1 & 1 & $90.56 \pm 2.95$ & 90.05 \\
\hline 20 & -1 & -1 & -1 & -1 & -1 & -1 & -1 & -1 & -1 & -1 & $60.31 \pm 3.20$ & 63.93 \\
\hline
\end{tabular}

Each experiment was repeated three times, and all of the data were expressed as means \pm standard deviations. $X_{1}, X_{2}, X_{3}, X_{4}, X_{5}, X_{6}, X_{7}, X_{8}, X_{9}$, and $X_{10}$ represent peptone $(\mathrm{g} / \mathrm{L})$, corn powder $(\mathrm{g} / \mathrm{L})$, starch $(\mathrm{g} / \mathrm{L})$, soybean meal $(\mathrm{g} / \mathrm{L}), \mathrm{KH}_{2} \mathrm{PO}_{4}(\mathrm{~g} / \mathrm{L}),\left(\mathrm{NH}_{4}\right)_{2} \mathrm{SO}_{4}(\mathrm{~g} / \mathrm{L})$, incubation temperature $\left({ }^{\circ} \mathrm{C}\right)$, initial $\mathrm{pH}$, incubation time $(\mathrm{h})$, and inoculum size (\%).

2.6. Response Surface Methodology. Through the PlackettBurman design experiment, the significant variables were selected as follows: soybean meal, corn powder, and incubation temperature. After that, the Box-Behnken design, a type of response surface methodology, was used to determine the optimum level of these selected variables for producing sublancin 168 as highly as possible. With the help of the statistical software package "Design Expert 8.0.5b" (Shanghai TechMax Co., Ltd., Shanghai, China), the experimental design was analyzed and 15 experiments in all were formulated. The central values of every variable were coded 0 . The maximum and minimum ranges of the variables were set up, and the whole experiment program in terms of their coded and actual values is shown in Table 5. In all trials the response values $(Y)$ were the average of three replicates.

2.7. Batch Fermentation in a $5 \mathrm{~L}$ Bioreactor. To investigate the behaviour of sublancin 168 accumulation, batch fermentations were conducted in a $5 \mathrm{~L}$ bioreactor (NBS Co., USA). The prepared seed culture was inoculated $(2 \%, \mathrm{v} / \mathrm{v})$ into the optimal medium with an initial $\mathrm{pH}$ 7.0. According to the preexperiment results (data not shown), the bioreactor was operated with optimized temperature, airflow at $1.5 \mathrm{vvm}$, and stirring at $500 \mathrm{rpm}$, and the $\mathrm{pH}$ was uncontrolled during fermentation. 
TABLE 4: Experimental design and corresponding response of steepest ascent.

\begin{tabular}{lcccc}
\hline Experiment number & Corn powder $(\mathrm{g} / \mathrm{L})$ & Soybean meal $(\mathrm{g} / \mathrm{L})$ & Incubation temperature $\left({ }^{\circ} \mathrm{C}\right)$ & Yield $(\mathrm{mg} / \mathrm{L})$ \\
\hline 0 & 12 & 8 & 25 & $72.7 \pm 2.97$ \\
$0+1 \Delta$ & 16 & 12 & 27 & $80.3 \pm 4.11$ \\
$0+2 \Delta$ & 20 & 16 & 31 & $89.5 \pm 2.42$ \\
$0+3 \Delta$ & 24 & 20 & 33 & $117.5 \pm 3.58$ \\
$0+4 \Delta$ & 28 & 24 & 35 & $122.6 \pm 1.64$ \\
$0+5 \Delta$ & 32 & 28 & $109.0 \pm 4.17$ \\
\hline
\end{tabular}

Each experiment was repeated three times, and all of the data were expressed as means \pm standard deviations.

TABLE 5: Experimental design and results of Box-Behnken optimization experiment.

\begin{tabular}{|c|c|c|c|c|c|}
\hline \multirow{2}{*}{ Trials } & \multirow{2}{*}{$X_{1}$} & \multirow{2}{*}{$X_{2}$} & \multirow{2}{*}{$X_{3}$} & \multicolumn{2}{|c|}{ Yield (mg/L) } \\
\hline & & & & Observed & Predicted \\
\hline 1 & 22.00 & 24.00 & 36.00 & $73.66 \pm 1.62$ & 73.43 \\
\hline 2 & 34.00 & 28.00 & 32.00 & $86.35 \pm 2.78$ & 86.78 \\
\hline 3 & 28.00 & 28.00 & 28.00 & $77.37 \pm 1.83$ & 77.57 \\
\hline 4 & 28.00 & 24.00 & 32.00 & $124.39 \pm 1.92$ & 124.15 \\
\hline 5 & 22.00 & 28.00 & 32.00 & $71.97 \pm 1.26$ & 71.81 \\
\hline 6 & 34.00 & 24.00 & 28.00 & $94.52 \pm 3.73$ & 94.75 \\
\hline 7 & 28.00 & 28.00 & 36.00 & $92.97 \pm 5.06$ & 93.37 \\
\hline 8 & 28.00 & 20.00 & 28.00 & $115.92 \pm 2.90$ & 115.53 \\
\hline 9 & 22.00 & 20.00 & 32.00 & $86.35 \pm 2.72$ & 85.93 \\
\hline 10 & 28.00 & 24.00 & 32.00 & $124.22 \pm 1.24$ & 124.15 \\
\hline 11 & 28.00 & 24.00 & 32.00 & $123.85 \pm 1.63$ & 124.15 \\
\hline 12 & 34.00 & 20.00 & 32.00 & $92.67 \pm 3.56$ & 93.37 \\
\hline 13 & 34.00 & 24.00 & 36.00 & $90.80 \pm 2.90$ & 90.82 \\
\hline 14 & 22.00 & 24.00 & 28.00 & $91.98 \pm 3.72$ & 91.95 \\
\hline 15 & 28.00 & 20.00 & 36.00 & $77.48 \pm 2.35$ & 77.28 \\
\hline
\end{tabular}

Each experiment was repeated three times, and all of the data were expressed as means \pm standard deviations. $X_{1}, X_{2}$, and $X_{3}$ represent corn powder $(\mathrm{g} / \mathrm{L})$, soybean meal, and incubation temperature $\left({ }^{\circ} \mathrm{C}\right)$, respectively.

2.8. Quantitative Determination of Sublancin 168 Content. Isolation and purification of sublancin 168 were carried out as previously described [1], with slight modification. The collected supernatant was made in $1 \mathrm{M} \mathrm{NaCl}$ and subjected to a hydrophobic interaction chromatography of $25 \mathrm{~mL}$ Toyopearl Butyl-650 column (Tosoh, Tokyo, Japan), and then a solution of $50 \mathrm{mM} \mathrm{NaAc}, \mathrm{pH} 4.0$, was used to wash down the sublancin. Subsequently the elution was made in 0.1 trifluoroacetic acid (TFA) and subjected to a semipreparative Zorbax 300SB-C8 column $(250 \times 9.4 \mathrm{~mm}, 5 \mu \mathrm{m}$ particle size, $300 \AA$ pore size) (Agilent, Englewood, CO) with a linear 0$60 \%$ acetonitrile gradient at a flow rate of $1.0 \mathrm{~mL} / \mathrm{min}$. The active fractions were collected and applied to an analytical Zorbax $300 \mathrm{SB}-\mathrm{C} 8$ column $(150 \times 4.6 \mathrm{~mm}, 5 \mu \mathrm{m}$ particle size, $300 \AA$ pore size) (Agilent, Englewood, CO) with the same conditions as the first step. The absorbances at $214 \mathrm{~nm}$, $254 \mathrm{~nm}$, and $280 \mathrm{~nm}$ were monitored. The concentration of purified sublancin 168 was determined by UV spectrophotometry $[13,14]$. Using purified sublancin as standard sample, the fermentation broths were applied to analytical Zorbax 300SB-C8 column to determine sublancin 168 concentrations with the method used in purification of sublancin 168 .
2.9. Statistics. During this study, each experiment was repeated three times, and all of the data were expressed as means \pm standard deviations.

\section{Results and Discussion}

3.1. Screening Optimal Carbon Sources and Nitrogen Sources. According to the fermentation result (data not shown) obtained by using the method reported in the literatures $[1,3]$, there is a no more than $60 \mathrm{mg}$ sublancin 168 from one liter bacterial culture. Thus, a new fermentation method with some different media and culture conditions is required to efficiently produce sublancin 168. As illustrated in Table 1, among the evaluations with different nitrogen sources, soybean meal showed an outstanding effect on the sublancin 168 production of $58.40 \mathrm{mg} / \mathrm{L}$, followed by peptone of $50.11 \mathrm{mg} / \mathrm{L}$. Urea had played an insignificant role on this peptide production. Among the different tested carbon sources, corn powder had a prominent effect on the sublancin 168 production of $67.66 \mathrm{mg} / \mathrm{L}$, followed by starch of $50.65 \mathrm{mg} / \mathrm{L}$, and glycerol had a sight effect on the yield of sublancin 168. Corn powder and soybean meal play an important role 
TABLE 6: Analysis of variances of the quadratic polynomial model.

\begin{tabular}{lcccc}
\hline Source & SS & DF & MS & $F$-value \\
\hline Model & 4776.00 & 9 & 530.67 & 2507.25 \\
Lack of fit & 0.90 & 3 & 0.30 & 3.92 \\
Pure error & 0.15 & 2 & 0.08 & 0.2100 \\
\hline Total & 4777.05 & 14 & & \\
\hline$R^{2}=0.9998, R_{\text {Adj }}^{2}=0.9993$, and $R_{\text {Pre }}^{2}=0.9998$. SS: sum of squares; DF: degrees of freedom; MS: mean square. &
\end{tabular}

in production improvement of interest products, such as Acinetobacter sp. $\mathrm{DNS}_{32}$ strain [15], B. subtilis WHK-Z12 spore [16], $\beta$-glucanase from $B$. subtilis ZJF-1A5 [17], and alkaline protease from Bacillus sp. RKY3 [18]. In this study, the results of screening optimal carbon sources and nitrogen sources suggested that corn powder and soybean meal were also important for B. subtilis 168 strains to efficiently produce sublancin 168 .

3.2. Plackett-Burman Design. The Plackett-Burman design was employed to evaluate the relative importance of cultivation parameters and different medium components (Figure 1). The main effect of each variable upon sublancin 168 production was evaluated as the difference made between both averages of measurements at the high level $(+1)$ and at the low level $(-1)$ correspondingly (Table 2 ). The data in Table 3 showed a wide variation from $60.31 \mathrm{mg} / \mathrm{L}$ to $121.38 \mathrm{mg} / \mathrm{L}$. The results suggested that higher productivity of sublancin 168 was achieved from medium optimization. The significant variables affecting sublancin 168 productivity are soybean meal, corn powder, and incubation temperature from the calculation of $t$-values and $P$ values (Table 2 and Figure 1). The incubation time from $28 \mathrm{~h}$ to $40 \mathrm{~h}$ and inoculum size from $1 \%$ to $3 \%$ play an insignificant role on sublancin 168 production.

Based on (1) and analyzed by using Minitab, a first-order model was fitted to the results obtained from the twenty experiments as the following equation:

$$
\begin{aligned}
Y(\mathrm{mg} / \mathrm{L})= & 95.56+1.17 X_{1}+13.60 X_{2}+1.60 X_{3}+10.54 X_{4} \\
& +1.60 X_{5}-0.48 X_{6}+5.55 X_{7}-0.96 X_{8} \\
& +0.19 X_{9}-1.17 X_{10},
\end{aligned}
$$

where $Y$ is the sublancin 168 production and $X_{1}, X_{2}, X_{3}, X_{4}$, $X_{5}, X_{6}, X_{7}, X_{8}, X_{9}$, and $X_{10}$ are the coded values of peptone, corn powder, starch, soybean meal, $\mathrm{KH}_{2} \mathrm{PO}_{4},\left(\mathrm{NH}_{4}\right)_{2} \mathrm{SO}_{4}$, incubation temperature, initial $\mathrm{pH}$, incubation time, and inoculum size, respectively. The goodness of the regression model was determined by the coefficient of determination $R^{2}$ whose value is $96.76 \%$ and suggests that only $3.24 \%$ of the total variation could not be explained by the model. Hence it was reasonable to take the regression model to analyse the tread in the response.

3.3. Steepest Ascent. Even though Plackett-Burman design allows for the rapid selection of the significant variables affecting productivity of sublancin 168, the optimum levels of the variables cannot be predicted by this method. The method of steepest ascent is a procedure for moving sequentially along the path of steepest ascent and in the direction of the maximum increase in the response. In order to move the variables rapidly to the general vicinity of the optimum levels, the path of steepest ascent was used to find the proper direction to change the variables by increasing the incubation temperature and the concentration of soybean meal and corn powder to improve the production of sublancin 168. The results showed that the sublancin 168 production reached a yield plateau during the fifth step (Table 4). Thus, these three variables were selected for further optimization.

As illustrated in Table 4, the sublancin 168 production did not further increase with the increase of concentrations of corn powder and soybean meal and the increase of temperature. For corn powder and soybean meal, the yield of sublancin 168 decreased from $122.6 \mathrm{mg} / \mathrm{L}$ to $109.0 \mathrm{mg} / \mathrm{L}$ when the concentrations of corn powder and soybean meal increased from $28 \mathrm{~g} / \mathrm{L}$ to $32 \mathrm{~g} / \mathrm{L}$ and from $24 \mathrm{~g} / \mathrm{L}$ to $28 \mathrm{~g} / \mathrm{L}$, respectively. The increase temperature from $33^{\circ} \mathrm{C}$ to $35^{\circ} \mathrm{C}$ may make the cells more difficult to biosynthesize sublancin 168 ; therefore, the sublancin production was not improved.

3.4. Box-Behnken Design. To determine the optimum levels of these important independent variables (soybean meal, corn powder, and incubation temperature) according to the above results, a 3-factor Box-Behnken design with 3 levels involving 3 replicates at center point was introduced to fit a second-order response surface. Table 5 shows the design matrix and the corresponding experimental data. The quadratic regression equations were obtained according to sublancin 168 production after the above results were analysed through standard analysis of variance (ANOVA). With the data of designed experiments, the polynomial regression model (in coded value) for sublancin 168 yield $Y$ was regressed only with respect to the significant factors and was shown as follows:

$$
\begin{aligned}
Y= & 124.15+5.07 X_{1}-5.51 X_{2}-5.63 X_{3}+2.06 X_{1} X_{2} \\
& +3.65 X_{1} X_{3}+13.47 X_{2} X_{3}-21.51 X_{1}^{2}-18.35 X_{2}^{2} \\
& -14.91 X_{3}^{2},
\end{aligned}
$$

where $Y$ predicates the sublancin 168 production, $X_{1}$ is corn powder, $X_{2}$ is soybean meal, and $X_{3}$ is incubation temperature.

Based on F-test and ANOVA using the SAS software package, the statistical significance of (3) was evaluated. As shown in Table 6, F-value of the model is 2507.25, and F-value 
TABLE 7: Results of regression analysis of the second-order polynomial model.

\begin{tabular}{lcccc}
\hline Factor & Coefficient estimate & Standard error & $F$-value & $P>F$ \\
\hline Intercept & 124.15 & 0.27 & 2507.25 & 963.01 \\
$X_{1}$ & 5.05 & 0.16 & 1130.53 & $<0.0001$ \\
$X_{2}$ & -5.47 & 0.16 & 1189.84 & $<0.0001$ \\
$X_{3}$ & -5.61 & 0.16 & 76.82 & $<0.0001$ \\
$X_{1} X_{2}$ & 2.02 & 0.23 & 251.72 & 0.0001 \\
$X_{1} X_{3}$ & 3.65 & 0.23 & 3449.98 & $<003$ \\
$X_{2} X_{3}$ & 13.51 & 0.23 & 8068.33 & $<0.0001$ \\
$X_{1}^{2}$ & -21.51 & 0.24 & 5847.41 & $<0.0001$ \\
$X_{2}^{2}$ & -18.31 & 0.24 & 3877.87 & $<0.0001$ \\
$X_{3}^{2}$ & -14.91 & 0.24 & $<0.0001$ \\
\hline
\end{tabular}

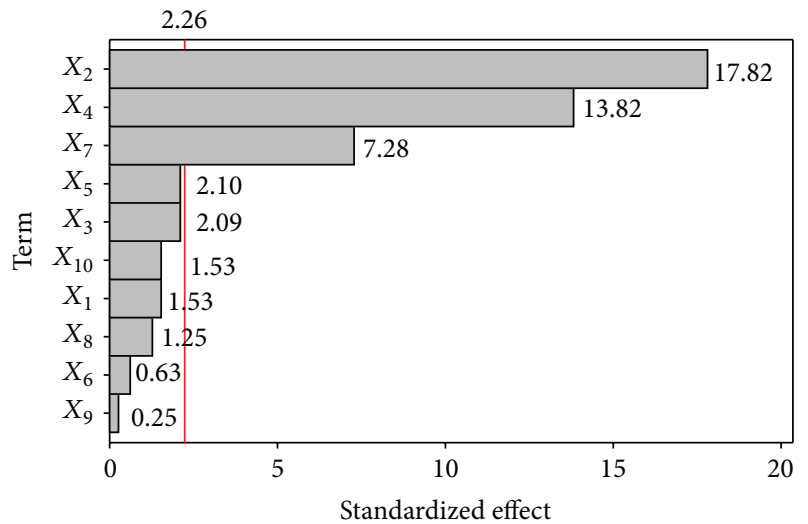

Figure 1: Pareto chart of standardized effects on sublancin 168 production. $X_{1}, X_{2}, X_{3}, X_{4}, X_{5}, X_{6}, X_{7}, X_{8}, X_{9}$, and $X_{10}$ are the coded values of peptone, corn powder, starch, soybean meal, $\mathrm{KH}_{2} \mathrm{PO}_{4}$, $\left(\mathrm{NH}_{4}\right)_{2} \mathrm{SO}_{4}$, incubation temperature, initial $\mathrm{pH}$, incubation time, and inoculum size, respectively. The chart has a vertical line (i.e., standardized effect $=1.886$ ) at the critical $t$-value for $\alpha$ of 0.20 . The bars are shown in order of the size of the effects, and the standardized effect of every term was displayed on the top of its corresponding bar.

for lack of fit is 3.92. The high $F$-value and nonsignificant lack of fit indicate that the model (3) is a good fit. This result indicates that the model used to fit response variable is significant $(P<0.0001)$ and adequate to represent the relationship between the responses and the independent variables. And the ANOVA (F-test) for this work is shown in Table 7 . The value of determination coefficient $R^{2}$ is 0.9996 , which means that we are able to explain $99.98 \%$ of results for sublancin 168 production using the calculated model. This result indicates that the model used to fit response variable is significant $(P<$ 0.0001 ) and adequate to represent the relationship between the responses and the independent variables. Meantime, the significance of the model was satisfactorily confirmed by the adjusted determination coefficient $\left(R_{\text {Adj }}^{2}=0.9993\right)$ and predicated determination coefficient $\left(R_{\text {pre }}^{2}=0.9998\right)$. Thus using this model to estimate the response trends is considered to be reasonable.

The model coefficient calculated from the regression analysis for each significant variable is shown in Table 7. Table 7 shows that the regression coefficients of individual linear, quadratic terms, and two cross products are statistically significant at $95 \%$ confidence level.

Three-dimensional (3D) response surface plots (Figure 2(a)) and two-dimensional (2D) contour plots (Figure 2(b)) are the graphical representations of the quadratic polynomial regression equation and usually illustrate the relationships between the experimental levels of each variable and corresponding response. The sublancin 168 production is shown in Figure 2 by $3 \mathrm{D}$ response surface plots and their respective $2 \mathrm{D}$ contour plots. Each figure reveals the interaction of two variables meanwhile the other is kept at zero level. In the 3D response surface plots and $2 \mathrm{D}$ contour plots as shown, the interaction exists within every pair of selected three factors and the effects are significant.

The model reveals that the corn powder concentration $\left(X_{1}\right)$, soybean meal concentration $\left(X_{2}\right)$, and temperature $\left(X_{3}\right)$ had a significant effect $(P<0.0001)$ on the sublancin 168 production $(Y)$. Positive coefficient of $X_{1}$ indicated a linear effect to increase, and negative coefficient of $X_{2}$ and $X_{3}$ suggested a linear effect to decrease. Meanwhile, quadratic term $X_{1}^{2}, X_{2}^{2}$, and $X_{3}^{2}$ had the negative effect. The graphs (Figure 2) illustrate the changes in the parameter modelled as the two factors move along those levels, while the other factor held constant at the central point. According to (3), it was predicted that a maximum sublancin 168 production of $125.88 \mathrm{mg} / \mathrm{L}$ appeared at $22.99 \mathrm{~g} / \mathrm{L}$ soybean meal and $28.49 \mathrm{~g} / \mathrm{L}$ corn powder, while temperature was held at $30.8^{\circ} \mathrm{C}$.

3.5. Model Verification in Shake-Flask. The availability of the regression model of the sublancin 168 production using the calculated optimal medium compositions and culture condition, namely, $22.99 \mathrm{~g} / \mathrm{L}$ soybean meal, $28.49 \mathrm{~g} / \mathrm{L}$ corn powder, and temperature at $30.8^{\circ} \mathrm{C}$, was validated with triplicate experiments. The mean maximal value of sublancin 168 production was $129.72 \mathrm{mg} / \mathrm{L}$, which agreed with the predicted value $(125.88 \mathrm{mg} / \mathrm{L})$ well. As a result, the model was considered to be accurate and reliable for predicting the sublancin 168 production by $B$. subtilis 168 . However, there is a certain gap between the yield and the theoretical value, which may be caused by some factors other than the medium that affect the yield of sublancin 168 but not investigated in this work. In this study, the yield of sublancin 168 by B. subtilis 168 was improved from low level of no more than $60 \mathrm{mg} / \mathrm{L}$ up to $129.72 \mathrm{mg} / \mathrm{L}$ in optimized medium. 

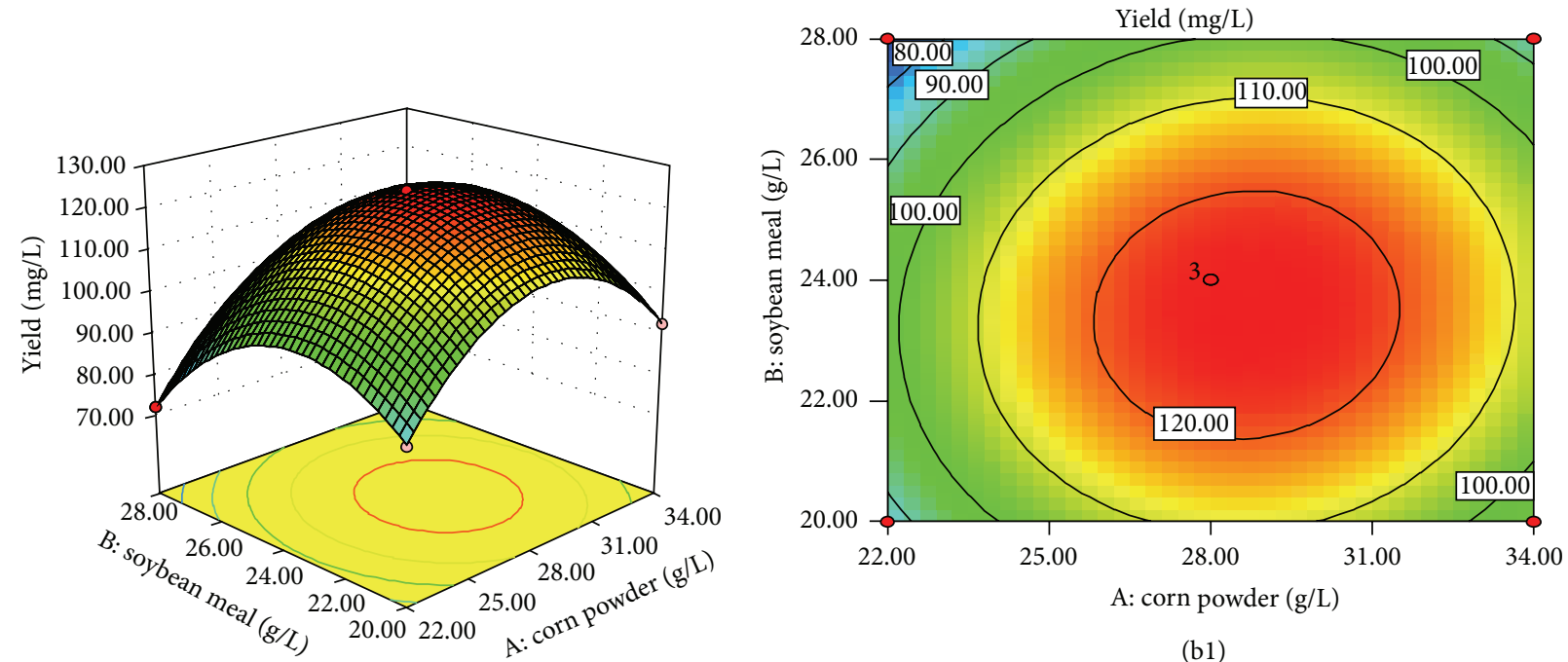

(a1)

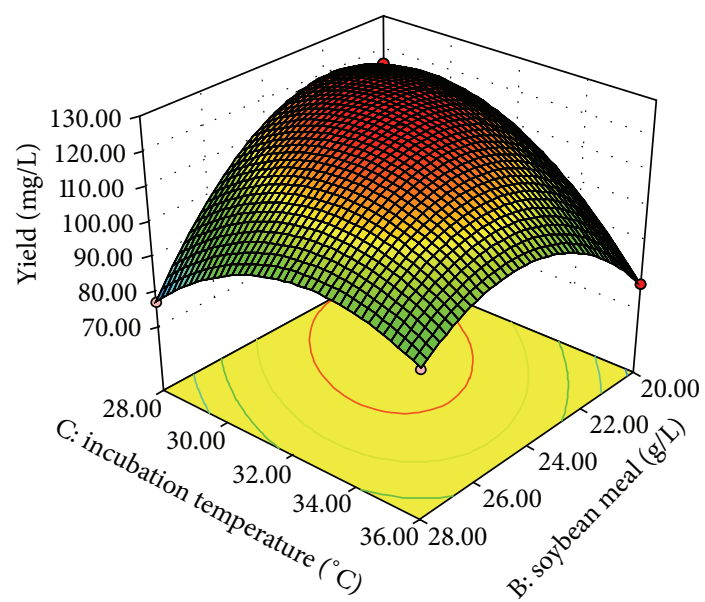

(a2)

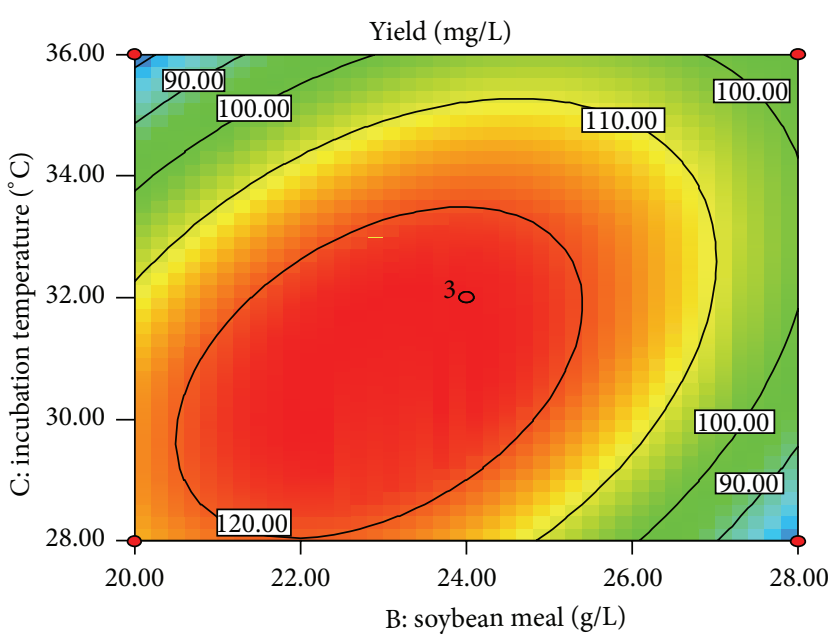

(b2)

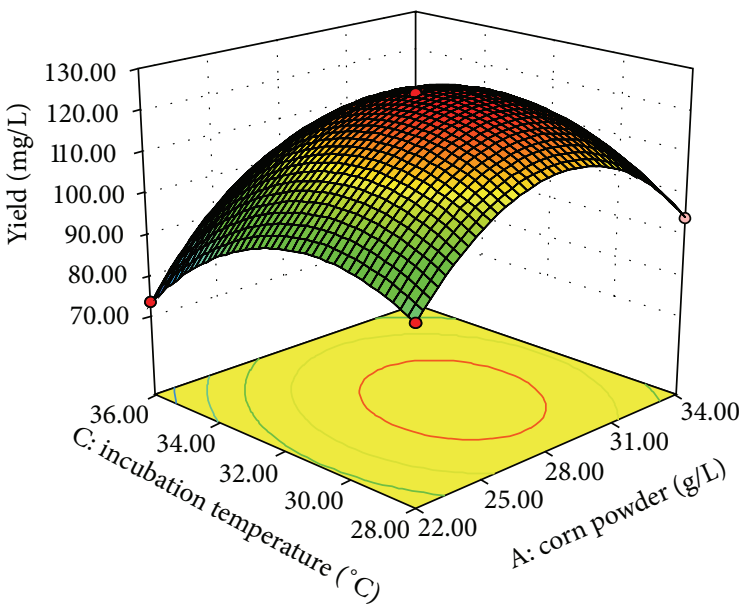

(a3)

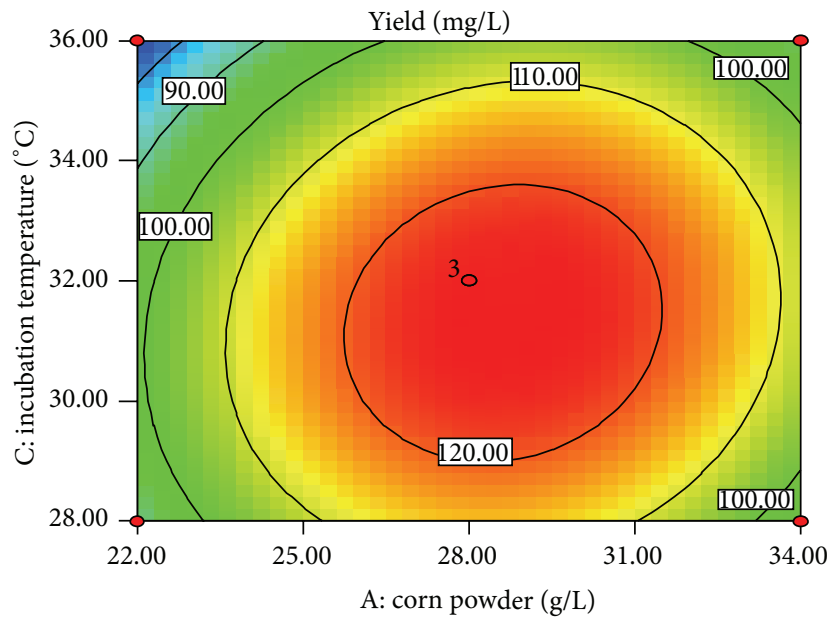

(b3)

(a)

(b)

Figure 2: 3D response surface curves (a) and 2D contour plots (b) predicting for sublancin 168 production by Bacillus subtilis 168 through optimization of variables. The interaction between $(\mathrm{a} 1, \mathrm{~b} 1)$ soybean meal and corn powder, $(\mathrm{a} 2, \mathrm{~b} 2)$ incubation temperature, and soybean meal and (a3, b3) incubation temperature and corn powder. 
3.6. Validation of the Model in Bioreactor. Using the optimal medium and temperature, sublancin 168 reached repeatable yield of $135.4 \mathrm{mg} / \mathrm{L}$ in bioreactor batch fermentation after about $48 \mathrm{~h}$ of cultivation. Although the temperature and medium components were coincident in flask and bioreactor fermentations, the yield of sublancin 168 in bioreactor fermentation $(135.4 \mathrm{mg} / \mathrm{L})$ was higher than that in the shake-flask culture $(129.72 \mathrm{mg} / \mathrm{L})$, probably mainly due to the differences of aeration conditions. Even so, the working conditions of the bioreactor require further optimizations in future experiments to furtherly improve sublancin yield.

3.7. Influence of Corn Powder and Soybean Meal on Sublancin 168 Production. In B. subtilis 168 , there are six proteins (SunI, SunA, SunT, BdbA, SunS, and BdbB) [2] taking part in biosynthesizing mature sublancin 168 , and the biosynthesis of sublancin 168 is controlled under a complex regulatory network that involves a minimum of five transcriptional regulators, including Abh, AbrB, Rok, YvrG, and YvrH [1921]. Corn powder and soybean meal are commonly substrates used by bacteria to produce enzymes and other secondary metabolites through fermentation. B. subtilis 168 possesses an ATP-dependent protein kinase which can be activated by several metabolites (fructose 1,6-diphosphate, gluconate-6$\mathrm{P}$, and 2-phosphoglycerate) when growing in the presence of corn powder. The activated protein kinase phosphorylates a seryl residue (ser-46) of HPr, a small phosphocarrier protein [22]. HPr probably have a direct or indirect control function on the complex regulatory network that regulates the biosynthesis of sublancin 168. Soybean meal is a highly concentrated source of protein [23] and can provide an excellent profile of amino acids for producing sublancin 168 .

\section{Conclusions}

As a summary, response surface methodology combined with Plackett-Burman design and steepest ascent enabled us to optimize the sublancin 168 yield produced by B. subtilis 168. The optimal combinations of culture parameters for maximum production of sublancin 168 were determined as corn powder $28.49 \mathrm{~g} / \mathrm{L}$, soybean meal $22.99 \mathrm{~g} / \mathrm{L}$, and incubation temperature $30.8^{\circ} \mathrm{C}$, and the maximum production of sublancin 168 was significantly improved from no more than $60 \mathrm{mg} / \mathrm{L}$ before optimization up to $129.72 \mathrm{mg} / \mathrm{L}$. To our knowledge, this is first report of statistical optimization for sublancin 168 production, which would provide some important parameters for large scale fermentation of this agent.

\section{Conflict of Interests}

The authors declare that there is no conflict of interests.

\section{Authors' Contribution}

Shengyue Ji and Weili Li contributed equally to this work.

\section{Acknowledgments}

This research was supported by the National Science and Technology R\&D Program of China (Grant no. 2011BAD28B05-3) and the Provincial Agriculture Special Fund Project of China (Grant no. 2011NYTT03).

\section{References}

[1] S. H. Paik, A. Chakicherla, and J. Norman Hansen, "Identification and characterization of the structural and transporter genes for, and the chemical and biological properties of, sublancin 168, a novel lantibiotic produced by Bacillus subtilis 168," The Journal of Biological Chemistry, vol. 273, no. 36, pp. 23134-23142, 1998.

[2] T. J. Oman, J. M. Boettcher, H. Wang, X. N. Okalibe, and W. A. van der Donk, "Sublancin is not a lantibiotic but an S-linked glycopeptide," Nature Chemical Biology, vol. 7, no. 2, pp. 78-80, 2011.

[3] S. Banerjee and J. N. Hansen, "Structure and expression of a gene encoding the precursor of subtilin, a small protein antibiotic," Journal of Biological Chemistry, vol. 263, no. 19, pp. 9508-9514, 1988.

[4] L.-W. Zhu, C.-C. Wang, R.-S. Liu, H.-M. Li, D.-J. Wan, and Y.J. Tang, "Actinobacillus succinogenes ATCC 55618 fermentation medium optimization for the production of succinic acid by response surface methodology," Journal of Biomedicine and Biotechnology, vol. 2012, Article ID 626137, 9 pages, 2012.

[5] P. Singh, S. S. Shera, J. Banik, and R. M. Banik, "Optimization of cultural conditions using response surface methodology versus artificial neural network and modeling of l-glutaminase production by Bacillus cereus MTCC 1305," Bioresource Technology, vol. 137, pp. 261-269, 2013.

[6] A. Abd Elrazak, A. C. Ward, and J. Glassey, "Response surface methodology for optimising the culture conditions for eicosapentaenoic acid production by marine bacteria," Journal of Industrial Microbiology and Biotechnology, vol. 40, no. 5, pp. 477-487, 2013.

[7] Z. Song, Q. Liu, H. Guo et al., “Tostadin, a novel antibacterial peptide from an antagonistic microorganism Brevibacillus brevis XDH," Bioresource Technology, vol. 111, pp. 504-506, 2012.

[8] W. Raza, W. Hongsheng, and S. Qirong, "Use of response surface methodology to evaluate the effect of metal ions $\left(\mathrm{Ca}^{2+}\right.$, $\left.\mathrm{Ni}^{2+}, \mathrm{Mn}^{2+}, \mathrm{Cu}^{2+}\right)$ on production of antifungal compounds by Paenibacillus polymyxa," Bioresource Technology, vol. 101, no. 6, pp. 1904-1912, 2010.

[9] Y. Wang, X. Fang, F. An, G. Wang, and X. Zhang, "Improvement of antibiotic activity of Xenorhabdus bovienii by medium optimization using response surface methodology," Microbial Cell Factories, vol. 10, article 98, 2011.

[10] J. He, Q. Zhen, N. Qiu et al., "Medium optimization for the production of a novel bioflocculant from Halomonas sp. V3a' using response surface methodology," Bioresource Technology, vol. 100, no. 23, pp. 5922-5927, 2009.

[11] R. L. Plackett and J. P. Burnam, "The design of optimum multifactorial experiments," Biometrika, vol. 33, no. 4, pp. 305325, 1946.

[12] D. C. Montgomeryd and G. C. Runger, Applied Statistics and Probability for Engineers, John Wiley \& Sons, New York, NY, USA, 3rd edition, 2002.

[13] H. Edelhoch, "Spectroscopic determination of tryptophan and tyrosine in proteins," Biochemistry, vol. 6, no. 7, pp. 1948-1954, 1967. 
[14] S. C. Gill and P. H. von Hippel, "Calculation of protein extinction coefficients from amino acid sequence data," Analytical Biochemistry, vol. 182, no. 2, pp. 319-326, 1989.

[15] Y. Zhang, Y. Wang, Z. G. Wang et al., "Optimization of fermentation medium for the production of atrazine degrading strain Acinetobacter sp. $\mathrm{DNS}_{32}$ by statistical analysis system," Journal of Biomedicine and Biotechnology, vol. 2012, Article ID 623062, 7 pages, 2012.

[16] Z.-M. Chen, Q. Li, H.-M. Liu et al., "Greater enhancement of Bacillus subtilis spore yields in submerged cultures by optimization of medium composition through statistical experimental designs," Applied Microbiology and Biotechnology, vol. 85, no. 5, pp. 1353-1360, 2010.

[17] X.-J. Tang, G.-Q. He, Q.-H. Chen, X.-Y. Zhang, and M. A. M. Ali, "Medium optimization for the production of thermal stable $\beta$-glucanase by Bacillus subtilis ZJF-1A5 using response surface methodology," Bioresource Technology, vol. 93, no. 2, pp. 175-181, 2004.

[18] L. V. A. Reddy, Y.-J. Wee, J.-S. Yun, and H.-W. Ryu, "Optimization of alkaline protease production by batch culture of Bacillus sp. RKY3 through Plackett-Burman and response surface methodological approaches," Bioresource Technology, vol. 99, no. 7, pp. 2242-2249, 2008.

[19] M. A. Strauch, B. G. Bobay, J. Cavanagh, F. Yao, A. Wilson, and Y. Le Breton, "Abh and AbrB control of Bacillus subtilis antimicrobial gene expression," Journal of Bacteriology, vol. 189, no. 21, pp. 7720-7732, 2007.

[20] M. Albano, W. K. Smits, L. T. Y. Ho et al., "The rok protein of Bacillus subtilis represses genes for cell surface and extracellular functions," Journal of Bacteriology, vol. 187, no. 6, pp. 2010-2019, 2005.

[21] M. Serizawa, K. Kodama, H. Yamamoto, K. Kobayashi, N. Ogasawara, and J. Sekiguchi, "Functional analysis of the YvrGHb two-component system of Bacillus subtilis: identification of the regulated genes by DNA microarray and Northern blot analyses," Bioscience, Biotechnology, and Biochemistry, vol. 69, no. 11, pp. 2155-2169, 2005.

[22] M. H. Saier Jr., S. Chauvaux, J. Deutscher, J. Reizer, and J. J. Ye, "Protein phosphorylation and regulation of carbon metabolism in Gram- negative versus Gram-positive bacteria," Trends in Biochemical Sciences, vol. 20, no. 7, pp. 267-271, 1995.

[23] G. L. Cromwell, C. C. Calvert, T. R. Cline et al., "Variability among sources and laboratories in nutrient analyses of corn and soybean meal. NCR-42 committee on swine nutrition. North central regional-42," Journal of Animal Science, vol. 77, no. 12, pp. 3262-3273, 1999. 

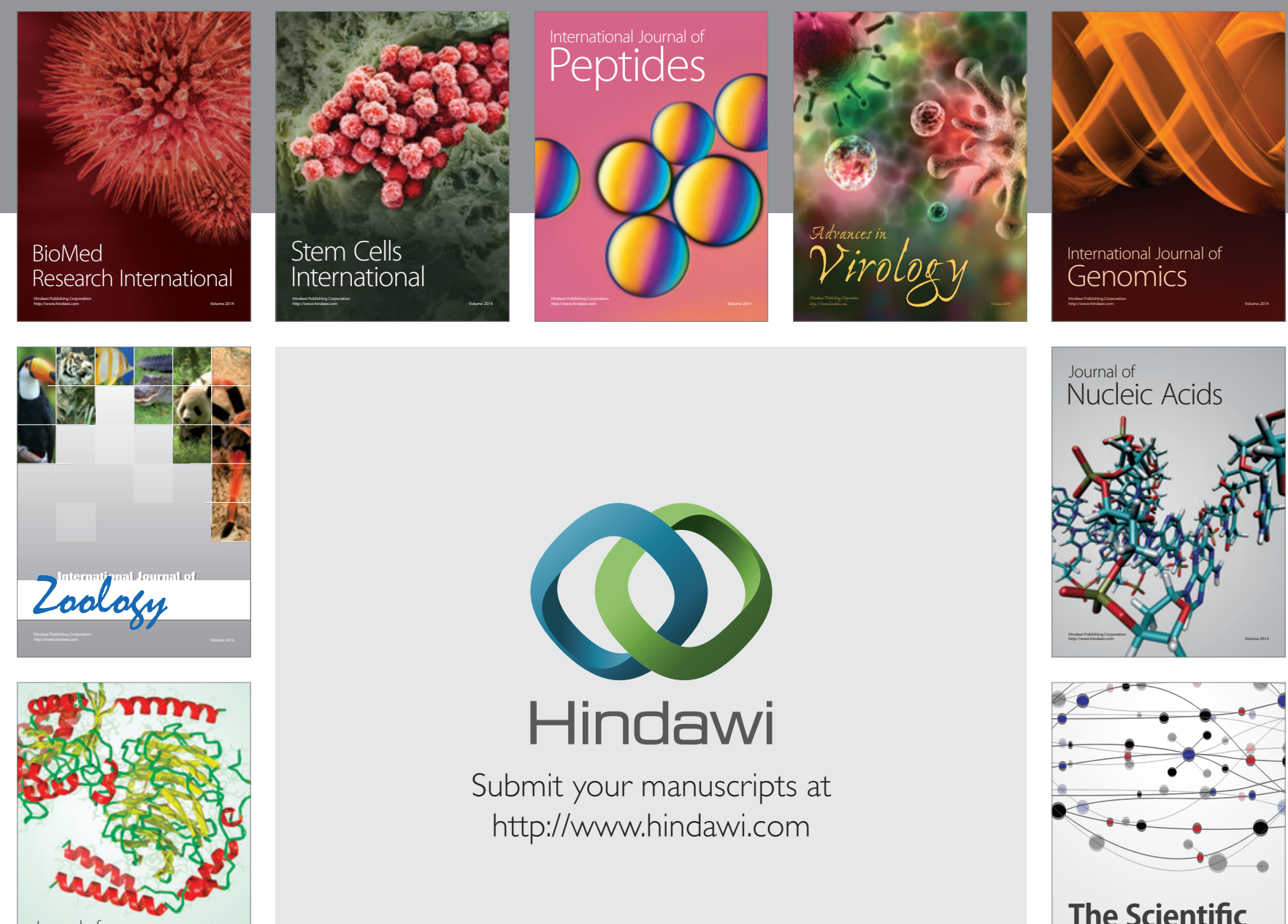

Submit your manuscripts at

http://www.hindawi.com

Journal of
Signal Transduction
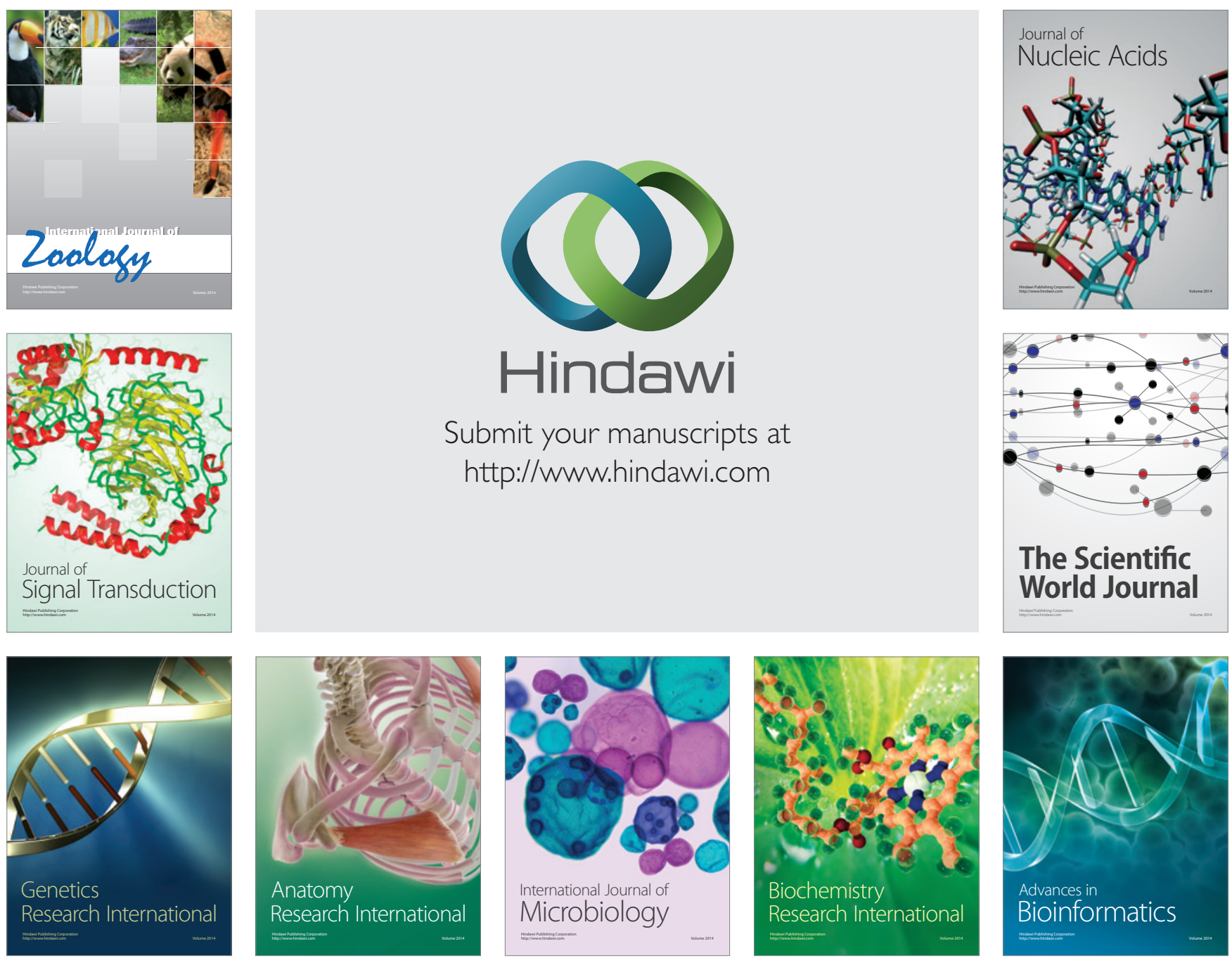

The Scientific World Journal
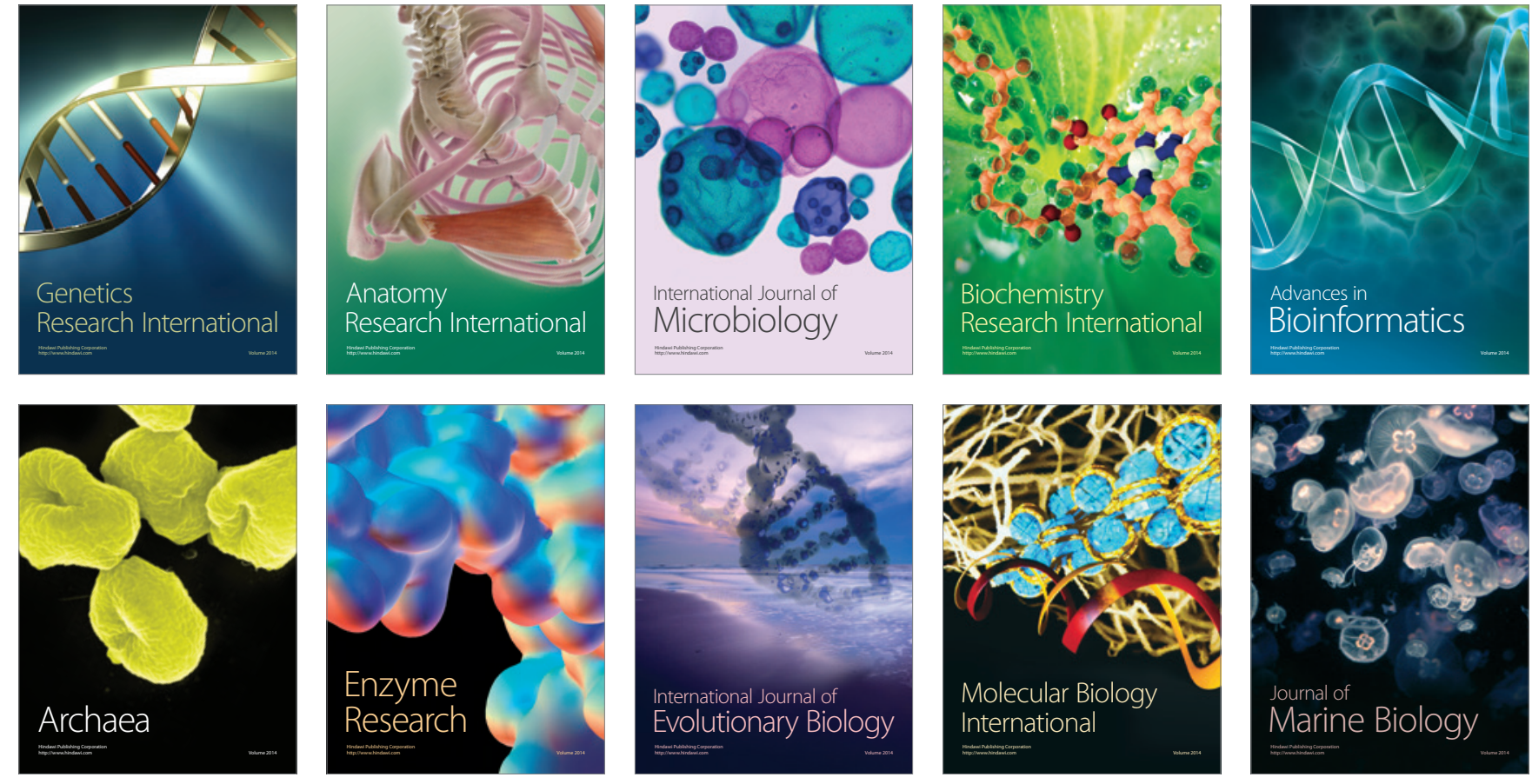